\title{
Implementation of a quantized line element in Klein-Gordon and Dirac fields
}

\author{
D. L. Bulathsinghala, K. A. I. L Wijewardena Gamalath \\ Department of Physics, University of Colombo, Colombo3, Sri Lanka \\ E-mail address: imalie@phys.cmb.ac.lk
}

\begin{abstract}
In this paper an ansatz that the anti-commutation rules hold only as integrated average over time intervals and not at every instant giving rise to a time-discrete form of Klein-Gordon equation is examined. This coarse-grained validation of the anti-commutation rules enables us to show that the relativistic energy-momentum relation holds only over discrete time intervals, fitting well with the timeenergy uncertainty relation. When this time-discrete scheme is applied to four vector notations in relativity, the line-element can be quantized and thereby how the physical attributes associated with time, space and matter can be quantized is sketched. This potentially enables us to discuss the Zeno's arrow paradox within the classical limit. As the solutions of the Dirac equation can be used to construct solutions to the Klein-Gordon equation, this temporal quantization rule is applied to the Dirac equation and the solutions associated with the Dirac equation under such conditions are interpreted. Finally, the general relativistic effects are introduced to a line-element associated with a particle in relativistic motion and a time quantized line-element associated with gravity is obtained.
\end{abstract}

Keyword: Time quantization; Klein-Gordon equation; Dirac equation; quantum gravity; energy-time uncertainty relation, continuity equation; probability densities; chronon, Zeno's arrow paradox; Zeno's Achilles and the tortoise paradox

\section{INTRODUCTION}

Although the time is treated as a continuous quantity both in quantum mechanics and relativity, many have suggested that a quantized time model may help to produce a theory of quantum gravity. Chronon, a quantum of time that is discrete and indivisible unit of time was proposed by Lévi in 1927 [1]. In the early days of quantum theory as a way to eliminate infinities from quantum field theory, the idea of a quantum spacetime, a generalization of the usual concept of spacetime in which some variables that ordinarily commute are assumed not to commute forming a different Lie algebra was proposed by Heisenberg and Ivanenko. Lorentz invariant discrete space-time was first published by Snyder in 1947 [1] using Lie algebra. Simplifying Snyder's Lie algebra, a quantum theory in which the time is a quantum variable with a discrete spectrum, consistent with special relativity was proposed by Yang in the same year [2]. Margenau in 1950 suggested that the chronon might be the time for light to travel the classical radius of an electron [3]. A prominent model was introduced by by Caldirola in 1980 giving a formula to obtain the value for the chronon, a quantization of the evolution in a 
system along its world line which is much longer than the Planck time, a universal quantization of time itself [4]. Since then various theorists have done further research into time quantization or the chronon model. Vaknin [4] proposed that the time is the result of the interaction of chronons in the time field and time asymmetry is generated as we observe them. In order to find time that will be independent of the choice of coordinates, an upper limit of measurable time form an event, back to the big bang singularity was proposed by Suchard [5]. Tifft put forward the idea that the redshifts of galaxies are quantized and could possibly explain an underlying time quantization scheme [6]. A more recent developments on time quantized models are based on a quasi-local invariant observable by Elze [7] and a perturbative approach to quantization by Barbero et.al,[8]. Kitada [9] had shown that quantum mechanical clock is equivalent to the relativistic classical clock. A quantize simple cosmological models was introduced by Halliwell [10] while Warner et.al [11] introduced the geometrodynamic quantization.

According to the Zeno's arrow paradox, generally thought to have been devised by ancient Greek philosopher Zeno of Ela, if everything is motionless at every instant, and time is entirely composed of many such instants, then motion is impossible. Further investigation into this paradox reveals that if a moving arrow is motionless or at rest for every temporal instant, then by comparing it with an arrow which is at rest for all time, one cannot distinguish between the two, at a given instant, which also suggests that motion must be an illusion. Contrary to the view that treats the observable quantities arising from four vector notation in relativity as conserved physical quantities at all instants, defining a conserved momentum at all instants is problematic as the very notion of the momentum requires a finite displacement over a finite time interval. Therefore, at a given instant, when conserved physical quantities associated with a moving body and that of a body at rest are compared, there appears to be no concrete evidence to suggest that one can be distinguished from the other. To resolve this problem, Russell [12] offered what is known as "at-at theory of motion", where it agrees that there can be no motion during a durationless instant, and all that is required for the motion is that the arrow be at one point at one time and at another point at another time. Lynds [13] suggesting a different solution argued that the whole notion of an instant is flawed and that there really aren't any instances, but intervals. The other notable efforts to resolve this paradox came from the assumption that the space is comprised of finite and discrete units, but the reconciliation of this view with the relativity, where space is being treated as a continuous quantity was problematic.

In quantum gravity, the quest of the research is to seek a description of gravity, or more precisely the principles governing gravity, in accordance with the principles of quantum mechanics. As our current understanding of gravity is based on the relativity proposed by Einstein, which is a formulation within the framework of classical physics, reconciliation requires that the classical view of space and time being continuous requires some radical changes. However, difficulties arise when one attempts to introduce the usual quantization techniques, as it becomes non-renormalizable and therefore cannot be used to make any meaningful predictions. As a direct consequence, theorists have taken up more radical approaches to the problem of quantum gravity, with the most popular among them being string theory and loop quantum gravity.

In this paper, the possibility of time quantization is considered as an intriguing question that may have relevance to quantum gravity as well as foundations of quantum mechanics. One way to investigate such questions in a more limited way, rather than within the framework of quantum field theory, is to return back to the simple relativistic quantum mechanics that predate field theory. Hence returning to the Klein-Gordon and Dirac equations, an ansatz, where anti- 
commutation rules hold only as integrated average over a time interval and not at every instant was examined. A first order inner-product solution to the Klein-Gordon equation was obtained by introducing this ansatz. This coarse-grained validation of the anti-commutation rules, when applied to the Klein-Gordon equation, giving a temporally quantized form of the Klein-Gordon equation shows that the energy-momentum relation holds only over discrete temporal intervals, consistent with the time-energy uncertainty phenomenon in quantum mechanics. This rule was used to show that the observable quantities associated with four-vector notation in relativity are only conserved within this time-discrete scheme. Thereby quantizing the line-element the Zeno's arrow paradox, Achilles and the tortoise paradox were potentially resolved. As the solutions of the Dirac equations are automatically solutions to the Klein-Gordon equation, how this timequantization scheme interprets the Dirac equation and its solutions are explored. This timediscrete scheme was extended to a line-element associated with gravity and how the ansatz presented in this paper could potentially be further investigated as a possible approach to quantum gravity is discussed.

\section{INNER PRODUCT FORM OF THE KLEIN-GORDON EQUATION}

The Schrödinger equation is not relativistically correct [14], as it does not contain space and time derivatives of the same order. Historically, the first attempt to construct a relativistic version of the Schrödinger equation began by applying the familiar quantization rules to the relativistic energy-momentum invariant $P^{\mu} P_{\mu}$. In Minkowskian spacetime this invariant,

$$
P^{\mu} P_{\mu}=g_{\mu v} P^{\mu} P^{v}=\left(\frac{E}{c}, \boldsymbol{p}\right) \cdot\left(\frac{E}{c},-\boldsymbol{p}\right)=\frac{E^{2}}{c^{2}}-p^{2}=(m c)^{2}
$$

where $P^{\mu}$ is the four momentum, $E$ is the energy, $\boldsymbol{p}$ is the three dimensional momentum, $m$ is the rest mass of the particle and $g_{\mu v}$ is the metric tensor defined as $(1,-1,-1,-1)$. In non-relativistic quantum mechanics, since the correspondence principle dictates that the momentum operator is associated with the spatial gradient $\left(\hat{p}_{i}=-i \hbar \partial_{i}\right)$, and the energy operator with the time derivative $\left(\hat{E}=i \hbar \partial_{t}\right)$ and if the wave function associated with the particle is $\psi(\boldsymbol{r}, t)$, then applying them to equation 1 , the Klein-Gordon equation $[15,16]$ can be obtained:

$$
\left(\nabla^{2}-\frac{1}{c^{2}} \frac{\partial^{2}}{\partial t^{2}}\right) \psi(\boldsymbol{r}, t)=\left(\frac{m c}{\hbar}\right)^{2} \psi(\boldsymbol{r}, t) .
$$

The inner product of Klein-Gordon equation, with a plane wave function can be written as:

$$
\left(\psi,\left(\nabla^{2}-\partial_{0}^{2}\right) \psi\right)=\left(\frac{m c}{\hbar}\right)^{2}(\psi, \psi)
$$

where, $\partial_{0}=\partial_{t} / c$. The Dirac equation was the first successful attempt to obtain an equation with both spatial and temporal derivatives of the same order, satisfying the wave equation. According 
to Dirac, the particle can exist in several distinct states with the same momentum but with different orientations of spin. Then the wave-function $\psi$ satisfying Klein-Gordon equation, given in equation 2, must have several components. It is not a scalar but a set of numbers each giving the probability amplitude to find the particle at a given place and in a given sub-state. The KleinGordon equation can be written in the form:

$$
\left(\nabla^{2}-\frac{1}{c^{2}} \frac{\partial^{2}}{\partial t^{2}}\right) \psi=\left(A_{i} \partial_{i}+i D \partial_{0}\right)\left(A_{i} \partial_{i}+i D \partial_{0}\right) \psi=\left(\frac{m c}{\hbar}\right)^{2} \psi
$$

To solve the above equation, satisfying $A_{i}^{2}=D^{2}=1$ and the anti-commutation conditions associated with cross terms, he found that for the exact solution, the terms $A_{i}$ and $D$ should represent $4 \times 4$ matrices known as the Dirac matrices:

$$
A_{i}=i \beta \alpha^{i}, \quad D=\beta, \quad\left\{\alpha^{i}, \alpha^{j}\right\}=2 \delta_{i j} \text { and }\left\{\alpha^{i}, \beta\right\}=0, \quad\left(\alpha^{i}\right)^{2}=\beta^{2}=I .
$$

Dirac equation can be written in more covariant form by introducing four matrices,

$$
\gamma^{\mu}=\left(\beta, \beta \alpha^{i}\right) \Rightarrow A_{i}=i \gamma^{i}, \quad D=\gamma^{0}
$$

For any general plane-wave solution, the following set of conditions

$$
\left(\partial_{\mu} \psi, \partial_{\nu} \psi\right)=-\left(\psi, \partial_{\mu} \partial_{\nu} \psi\right)=-\left(\psi, \partial_{\nu} \partial_{\mu} \psi\right)
$$

are satisfied $(\mu=0,1,2,3)$. With the above identity, one can define a general first-order innerproduct solution that satisfies the Klein-Gordon equation as,

$$
\begin{aligned}
\left(\left(i \gamma^{i \dagger} \partial_{i}+i \gamma^{0 \dagger} \partial_{0}\right) \psi,\left(i \gamma^{i} \partial_{i}+i \gamma^{0} \partial_{0}\right) \psi\right) & =\left(\psi,-\gamma^{i} \gamma^{i} \partial_{i}^{2} \psi\right)+\left(\psi,-\gamma^{0} \gamma^{0} \partial_{0}{ }^{2} \psi\right) \\
& -\frac{1}{2}\left\{\gamma^{i}, \gamma^{j}\right\}\left(\psi, \partial_{i} \partial_{j} \psi\right)-\left\{\gamma^{0}, \gamma^{i}\right\}\left(\psi, \partial_{0} \partial_{i} \psi\right)=\left(\frac{m c}{\hbar}\right)^{2}(\psi, \psi)
\end{aligned}
$$

where the four gamma matrices satisfy,

$$
\gamma^{0 \dagger}=\gamma^{0} ; \quad \gamma^{i \dagger}=-\gamma^{i} ; \quad \gamma^{\mu \dagger} \gamma^{\mu}=I ; \quad\left\{\gamma^{\mu}, \gamma^{v}\right\}=0(\mu \neq v)
$$

As the cross terms associated with anti-commutation rules for gamma-matrices vanish, for every temporal instance,

$$
\left(i \gamma^{\mu \dagger} \partial_{\mu} \psi, i \gamma^{\mu} \partial_{\mu} \psi\right)=\left(\psi,\left(\partial_{i}^{2}-\partial_{0}^{2}\right) \psi\right)=\left(\frac{m c}{\hbar}\right)^{2}(\psi, \psi)
$$

the above equation satisfy the Klein-Gordon equation for every instant of time. 


\section{A TEMPORALLY QUANTIZED FORM OF KLEIN-GORDON EQUATION}

In the general inner-product solution of the Klein-Gordon equation obtained, the cross terms associated with anti-commutation rules vanish for every temporal instance. On the other hand if a rule can be is introduced such that at least one or a few of the cross terms in equations 7 vanish, only when integrated over a periodic temporal interval, then the solution of this second order relativistic wave equation is said to be time-discrete or temporally quantized. An ansatz that a particle in relativistic motion is associated with two normalized complementary plane waves $\Psi$ such that, its temporal derivative is associated with a plane wave $\psi$ and its spatial derivatives are associated with a complementary wave $\psi_{c}$ is introduced [17]:

$$
\nabla^{2} \psi_{c}-\frac{1}{c^{2}} \frac{\partial^{2}}{\partial t^{2}} \psi=\partial_{i}^{2} \psi_{c}-\partial_{0}^{2} \psi=\left(\frac{m c}{\hbar}\right)^{2} \psi
$$

Then the inner product of Klein Gordon equation given in equation 10 can be written as

$$
\left(\left(i \gamma^{i \dagger} \partial_{i} \psi_{c}+i \gamma^{0 \dagger} \partial_{0} \psi\right),\left(i \gamma^{i} \partial_{i} \psi_{c}+i \gamma^{0} \partial_{0} \psi\right)\right)=\left(\frac{m c}{\hbar}\right)^{2}(\psi, \psi)
$$

Expanding the above equation

$$
\begin{gathered}
-\gamma^{i 2}\left(\psi_{c}, \partial_{i}^{2} \psi_{c}\right)-\gamma^{02}\left(\psi, \partial_{0}^{2} \psi\right)-\frac{1}{2}\left(\gamma^{i} \gamma^{j}+\gamma^{j} \gamma^{i}\right)\left(\psi_{c}, \partial_{i} \partial_{j} \psi_{c}\right) \\
\left(\left(\gamma^{0 \dagger} \partial_{0} \psi, \gamma^{i} \partial_{i} \psi_{c}\right)+\left(\gamma^{i \dagger} \partial_{i} \psi_{c}, \gamma^{0} \partial_{0} \psi\right)\right)=\left(\frac{m c}{\hbar}\right)^{2}(\psi, \psi) \\
\left(\psi_{c}, \partial_{i}^{2} \psi_{c}\right)-\left(\psi, \partial_{0}^{2} \psi\right)+\left(\left(\gamma^{0 \dagger} \partial_{0} \psi, \gamma^{i} \partial_{i} \psi_{c}\right)+\left(\gamma^{i \dagger} \partial_{i} \psi_{c}, \gamma^{0} \partial_{0} \psi\right)\right)=\left(\frac{m c}{\hbar}\right)^{2}(\psi, \psi)
\end{gathered}
$$

As $\psi$ and $\psi_{c}$ are complementary plane waves, by assigning two sets of complementary planewave solutions $\psi=e^{ \pm i(\omega t-\boldsymbol{k} \cdot \boldsymbol{r})}$ and $\psi_{c}=\psi^{*}$

$$
\left(\left(\gamma^{0 \dagger} \partial_{0} \psi, \gamma^{i} \partial_{i} \psi_{c}\right)+\left(\gamma^{i \dagger} \partial_{i} \psi_{c}, \gamma^{0} \partial_{0} \psi\right)\right)=\mp \frac{2 i \gamma^{0} \gamma^{i} k_{i} \omega}{c} \sin 2 \Omega \neq 0 \quad(\Omega=\omega t-\boldsymbol{k} \cdot \boldsymbol{r})
$$

and the anti-commutation hold only for discrete time instants corresponding to $\Omega=n \pi / 2$ where $n$ is a positive integer or when integrated over a temporal interval corresponding to an angular interval of $\pi$ radians $\left(\int_{\pi} \sin 2 \Omega d \Omega=0\right)$. However, in four-vector notation for all the terms associated with equation 10 , an invariant differential $\Omega=K^{\mu} X_{\mu}=(\omega t-\boldsymbol{k} \cdot \boldsymbol{r})=\omega_{0} \tau$, where $K^{\mu}$ is the four wave vector and $X^{\mu}$ the four position vector in a period of $\pi$ is chosen such that 


$$
\int_{\pi}\left(\left(\gamma^{0 \dagger} \partial_{0} \psi, \gamma^{i} \partial_{i} \psi_{c}\right)+\left(\gamma^{i \dagger} \partial_{i} \psi_{c}, \gamma^{0} \partial_{0} \psi\right)\right) d \Omega=0
$$

The anti-commutation between $\gamma^{i} \partial_{i} \psi_{c}$ and $\gamma^{0} \partial_{0} \psi$ now holds, when integrated over a periodic interval $\pi$. Thus, taking the integrated average of the inner-product equation 13, with respect to invariant differential $d \Omega$ over a periodic temporal interval $\pi$ :

$$
\frac{1}{\pi} \int_{\pi}\left(\left(\psi_{c}, \partial_{i}^{2} \psi_{c}\right)-\left(\psi, \partial_{0}^{2} \psi\right)\right) d \Omega+\frac{1}{\pi} \int_{\pi}\left(\left(\gamma^{0 \dagger} \partial_{0} \psi, \gamma^{i} \partial_{i} \psi_{c}\right)+\left(\gamma^{i \dagger} \partial_{i} \psi_{c}, \gamma^{0} \partial_{0} \psi\right)\right) d \Omega=\frac{1}{\pi}\left(\frac{m c}{\hbar}\right)^{2}(\psi, \psi) \int_{\pi} d \Omega
$$

leads to

$$
\left(\psi_{c}, \partial_{i}^{2} \psi_{c}\right)-\left(\psi, \partial_{0}^{2} \psi\right)=\left(\frac{m c}{\hbar}\right)^{2}(\psi, \psi)
$$

For plane waves selecting $\psi_{c}$ and $\psi$ such that

$$
\partial_{i}^{2} \psi_{c}=-k^{2} \psi_{c}, \quad \partial_{0}^{2} \psi=-(E / \hbar c)^{2} \psi
$$

and $\left(\psi_{c}, \psi_{c}\right)=1=(\psi, \psi)$ the relativistic energy-momentum invariant

$$
-k^{2}+\left(\frac{E}{\hbar c}\right)^{2}=\left(\frac{m c}{\hbar}\right)^{2} \Rightarrow E^{2}-p^{2} c^{2}=m^{2} c^{4}
$$

can be obtained. As the angular periodic interval $\pi$ corresponds to a temporal-interval, a temporally quantized form of the Klein-Gordon equation associated with two complementary waves is obtained which satisfies the energy-momentum relation when one takes the integrated average over discrete time intervals corresponding to an angle of $\pi$ radians given by:

$$
\frac{1}{\pi} \int_{\pi}\left(\left(i \hbar \gamma^{i \dagger} \partial_{i} \psi_{c}+i \hbar \gamma^{0 \dagger} \partial_{0} \psi\right),\left(i \hbar \gamma^{i} \partial_{i} \psi_{c}+i \hbar \gamma^{0} \partial_{0} \psi\right)\right) d \Omega=\frac{1}{\pi} \int_{\pi}(m c)^{2}(\psi, \psi) d \Omega
$$

or at angular instants corresponding to $\Omega=n \pi / 2$.

A first order time-quantized form of Dirac equation with complementary waves can be obtained as

$$
i \hbar \gamma^{\mu} \partial_{\mu} \psi=\left(i \hbar \gamma^{0} \partial_{0} \psi, i \hbar \gamma^{i} \partial_{i} \psi_{c}\right)=m c \psi
$$


For complementary plane waves $\psi=e^{ \pm i(\omega t-\boldsymbol{k} \cdot \boldsymbol{r})}$ and $\psi_{c}=\psi^{*}$ equation $21 \mathrm{read}$ :

$$
E \psi \mp \alpha^{i} p_{i} c \psi^{*}=\beta m c^{2} \psi
$$

\section{QUANTIZATION OF THE LINE INTERVAL}

In the theory of relativity, a four-vector $A^{\mu}(\mu=0,1,2,3)$ is a four dimensional vector in Minkowski space-time defined as a quantity which transforms under Lorentz transformations in the same way as coordinates of a point $\left(x_{0}, x_{1}, x_{2}, x_{3}\right)$, giving rise to a scalar product which is invariant under Lorentz transformation. Starting from the temporally quantized solution to the Klein-Gordon equation given in equation 17 and dividing by $\mathrm{m}^{2}$ :

$$
\begin{aligned}
& \frac{1}{\pi m^{2}} \int_{\pi}\left(\left(i \hbar \gamma^{i \dagger} \partial_{i} \psi_{c}+i \hbar \gamma^{0 \dagger} \partial_{0} \psi\right),\left(i \hbar \gamma^{i} \partial_{i} \psi_{c}+i \hbar \gamma^{0} \partial_{0} \psi\right)\right) d \Omega=\frac{1}{\pi} \int_{\pi} c^{2}(\psi, \psi) d \Omega . \\
& -(\gamma u)^{2}+(\gamma c)^{2}=c^{2}
\end{aligned}
$$

where $\gamma=d t / d \tau$. Writing the square of three dimensional velocity as $u^{2}=(d r / d t)^{2}$ and multiplying by $(d \tau)^{2}$ in the above equation,

$$
(c d t)^{2}-(d r)^{2}=(c d \tau)^{2}=d X^{\mu} d X_{\mu}=d s^{2}
$$

The square of the line-element associated with a particle in relativistic motion, which is timediscrete is obtained. Thus equation 25 holds only at discrete time instants or over discrete-time intervals when averaged. Using this time-discrete line-element, a time-discrete differential fourposition vector can be obtained:

$$
d X^{\mu}=(c d t, d \boldsymbol{r})=(c d t, d x, d y, d z)
$$

Therefore the spacetime differential elements, namely time and space are time-discrete or timequantized, over an interval $\Delta t=\pi / \omega$ where $\omega=\gamma m c^{2} / \hbar$ whose components are also timediscrete. By integrating the terms in equation 26 the four-position vector,

$$
X^{\mu}=(c t, \boldsymbol{r})=(c t, x, y, z)
$$

whose components are also time-discrete are obtained. Multiplying equation 20 by $\hbar^{2}$ the fourmomentum vector, 


$$
P^{\mu}=(E / c, \boldsymbol{p})
$$

whose components, namely energy and momentum are time-quantized, over an interval $\Delta t=\pi / \omega$ are obtained. Hence, the above work suggests that the line-element associated with a particle in relativistic motion is time-discrete and also that the time, space and matter components associated with a particle in relativistic motion are time-discrete. Thereby the other observable quantities associated with time, space and matter components in four-vector notation are conserved only over discrete time-intervals or at discrete time instants in accordance with the principles of quantum mechanics. The time-quantum $\Delta t$ associated with this quantizationscheme depends on the angular frequency $\omega$ of relativistic particle's matter-wave. Further, the periodic angular interval $\pi$ which corresponds to a time-quantum $\Delta t$ over which the conservation of the observable quantities hold can be regarded as a chronon which can be expressed in terms of the total-energy of the particle:

$$
\Delta t=\frac{\pi}{\omega}=\frac{h}{2}\left(\frac{1}{\gamma m c^{2}}\right)
$$

Taking the limiting case when a particle is having the maximum energy is bounded by Planckenergy $E_{P}$, an absolute smallest time-quanta or chronon associated with a particle can be derived:

$$
(\Delta t)_{\min }=\frac{1}{2} \frac{h}{E_{P}}=\frac{1}{2} t_{P}
$$

where the Planck time $t_{P}=5.39106 \times 10^{-44} \mathrm{~s}$.

\section{ZENO'S PARADOXS}

Many centuries ago, a Greek philosopher Zeno of Ela proposed that a flying arrow is at rest at each point of time during its flight. Comparing this flying arrow with an arrow at rest as one cannot distinguish them apart at each point of time or instant, he argued that motion must be an illusion. Russell [12] in his "at-at theory of motion" agreed that there can be no motion during a duration-less instant and for the motion, the requirement is that the arrow must be at one point at one time and at another point at another time. However, a close investigation into his claim suggests that if one can define infinitely many instants in-between the two finite instants, then motion becomes impossible as the arrow now needs to be at rest at all such in between infinitely many instants. The very notion of motion then becomes impossible. Therefore unless there is an absolute minimum temporal separation between two consecutive instants where there are no further in-between instants, the "at-at theory" will collapse.

Let us now re-visit, the ansatz presented in this paper, that a particle in a relativistic motion is associated with two complementary waves which indicates that the energy-momentum relation holds over discrete time intervals or at discrete time instants, and thereby the observable physical quantities associated with four-vector notation are also conserved over discrete time intervals or 
at discrete time instants. Further, and most importantly, if one were to associate the existence of a particle with the conservation of its physical quantities associated with four-vector notation, then the present work suggests that a particle in motion can exist only at discrete time instants or over discrete time intervals when averaged. This suggests that it is not proper to state that the particle exists at all time. Thus, we have particle instants, conservation holding at these instants, or particle intervals conservation holding over these intervals, where the energy-momentum relation is satisfied, along with the conservations of the particle's physical quantities associated with the four-vector notation.

Contrary to Lynds' claim that there aren't really any instants but intervals, present interpretation, that the conservation of the observable physical quantities associated with fourvector notation are only held over discrete time-intervals when averaged or at discrete time instants, suggests that, it is the conservation of the observable physical quantities that are timediscrete, and not the time itself. Further, this scheme then prohibits us from defining a set of conserved physical quantities attributed to an arrow, or more precisely to a particle, at any given instant, but rather for discrete time-intervals and at discrete instants, thus the existence of the arrow or the particle then becomes discrete, enabling us to resolve the paradox within the notion of continuous time, consistent with classical mechanics.

Similarly, Zeno's Achilles and the tortoise paradox states, in order for Achilles to overtake the tortoise, he must reach infinite number of points, where tortoise has already been, thus he can only get ever so closer to the tortoise, but cannot overtake it. Thus, if space is infinitely divisible, according to this paradox, Achilles will have to perform infinite number of steps, before overtaking the tortoise. Investigated further, this paradox questions whether a finite continuum can be sub divided to give infinite number of positions, therein. Throughout the history, several solutions have been proposed to resolve this paradox, notable among them are the works by Aristotle [17], Thomas Aquinas [18], Bertrand Russell [12] and Hans Reichenbach [19]. However, the present interpretation, presented in this paper, clearly suggest that, one cannot keep sub dividing the space, infinitely many times, and expect Achilles or a particle to reach each of those infinitely many points at different instants, as the very definition of Achilles (or the particle) only exists at discrete time instants or over discrete time intervals. Therefore, it is not proper to state, that Achilles (or the particle) must traverse through all those infinitely many possible points consecutively (at different instants), before overtaking the tortoise, as the very existence of Achilles (or the particle) now has become discrete. That is, Achilles (or the particle) now cannot claim to exist at each consecutive points one after the other, and thus, we are also able to resolve this paradox within the notion of continuous time, consistent with classical mechanics.

Since the total energy associated with a particle in relativistic motion is conserved only over discrete time-intervals, a measurement of a particle energy content $E$ over its conserved discrete time-interval $\Delta t$ yields a classical product between them:

$$
E \Delta t=\left(\gamma m c^{2}\right)\left(\frac{h}{2 \gamma m c^{2}}\right)=\frac{h}{2}
$$


In other words, the time-discrete conservation of energy, give rise to a type of relativistic timeenergy uncertainty. However, based on the interpretation given for uncertainty principles in quantum mechanics, the two observables associated in an uncertainty relation must arise from two operators which satisfy the canonical-commutation rule. As time is not considered a quantum mechanical operator there was some confusion with regard to the time-energy uncertainty relation in quantum mechanics. Nevertheless, in 1945, Mandelshtam and Tamm derived a non-relativistic time-energy uncertainty [20] for a quantum system in a stationary state, taking into account the life-time of the state to change its expectation value appreciably.

The confusion related to time not being considered a quantum mechanical operator does not arise with the time-discrete scheme examined in this paper, as the terms in the time-energy uncertainty relation to a particle's total energy and a discrete time-interval over which its energy is conserved are related. This suggests that the time-energy uncertainty relation is not a statement related to the observer-effect or the measurement-disturbance based on the precision of a measurement, but perhaps, a fundamental statement arising from this time-discrete scheme.

\section{TIME QUANTIZED FORM OF SPINORS}

For a particle with a particular momentum $\boldsymbol{p}$ and energy $E$, the wave function can be written in terms of a constant spinor $u$ as

$$
\Psi(\boldsymbol{r}, t)=u \psi(\boldsymbol{r}, t)
$$

The time-quantized form of Dirac equation given in equation 22, then becomes an equation for $u$ only,

$$
\left(E-\beta m c^{2}\right) u=\alpha^{i} p_{i} c u
$$

One possible set of $\alpha^{i}$ and $\beta$ is

$$
\alpha^{i}=\left(\begin{array}{cc}
0 & \sigma_{i} \\
\sigma_{i} & 0
\end{array}\right) ; \quad \beta=\left(\begin{array}{cc}
I & 0 \\
0 & -I
\end{array}\right)
$$

where $\sigma_{i} \mathrm{~s}$ are the Pauli spin matrices:

$$
\sigma_{1}=\left(\begin{array}{ll}
0 & 1 \\
1 & 0
\end{array}\right), \sigma_{2}=\left(\begin{array}{cc}
0 & -i \\
i & 0
\end{array}\right), \sigma_{3}=\left(\begin{array}{cc}
1 & 0 \\
0 & -1
\end{array}\right)
$$

Equation 33 written in full is, 


$$
\left(\begin{array}{cccc}
E_{-} & 0 & c p_{3} & c p_{-} \\
0 & E_{-} & c p_{+} & -c p_{3} \\
c p_{3} & c p_{-} & E_{+} & 0 \\
c p_{+} & -c p_{3} & 0 & E_{+}
\end{array}\right)\left(\begin{array}{l}
u_{1} \\
u_{2} \\
u_{3} \\
u_{4}
\end{array}\right)=\left(\begin{array}{cc}
E-m c^{2}\left(\begin{array}{cc}
1 & 0 \\
0 & 1
\end{array}\right) & -\sigma^{i} p_{i} c \\
-\sigma^{i} p_{i} c & E+m c^{2}\left(\begin{array}{ll}
1 & 0 \\
0 & 1
\end{array}\right)
\end{array}\right)\left(\begin{array}{l}
u_{A} \\
u_{B}
\end{array}\right)=0
$$

where $u=\left(\begin{array}{l}u_{A} \\ u_{B}\end{array}\right), \quad u_{A}=\left(\begin{array}{l}u_{1} \\ u_{2}\end{array}\right), \quad u_{B}=\left(\begin{array}{l}u_{3} \\ u_{4}\end{array}\right), E_{ \pm}=E \pm m c^{2}, p_{ \pm}=p_{1} \pm i p_{2}$.

Thus for a given $\boldsymbol{p}$ and energy $E= \pm \sqrt{p^{2} c^{2}+m^{2} c^{4}}$, there are four independent solutions of equation 36. By making a choice of $u_{A}=\left(\begin{array}{l}1 \\ 0\end{array}\right)$ or $\left(\begin{array}{l}0 \\ 1\end{array}\right)$ and $u_{B}=\left(\begin{array}{l}1 \\ 0\end{array}\right)$ or $\left(\begin{array}{l}0 \\ 1\end{array}\right)$ the wave functions in normalized forms are

$$
\Psi_{1}=N\left(\begin{array}{c}
1 \\
0 \\
c p_{3} / E_{+} \\
c p_{+} / E_{+}
\end{array}\right) \psi ; \quad \Psi_{2}=N\left(\begin{array}{c}
0 \\
1 \\
c p_{-} / E_{+} \\
-c p_{3} / E_{+}
\end{array}\right) \psi ; \quad \Psi_{3}=N\left(\begin{array}{c}
c p_{3} / E_{-} \\
c p_{+} / E_{-} \\
1 \\
0
\end{array}\right) \psi ; \quad \Psi_{4}=N\left(\begin{array}{c}
c p_{-} / E_{-} \\
-c p_{3} / E_{-} \\
0 \\
1
\end{array}\right) \psi
$$

Where $N=\sqrt{E+m c^{2}}$ is a normalized factor with $\Psi^{\dagger} \Psi=2 E$. The first two solutions in equation 37 are associated with spin-up and spin-down solutions of positive-energy particles, whereas the third and fourth solutions are associated with spin-up and spin-down negative-energy particles. This enabled Dirac to predict the existence of an anti-electron or the positron in 1931 [14]. Using the conventional Feynman-Stueckelberg interpretation [15], where a negative energy solution with $E<0$ represents a negative energy particle, traveling backwards in time, or equivalently, a positive energy particle going forward in time, the anti-particle states can be defined by just flipping the signs of $E$ and $p$. Thus the third and fourth solution of equation 37 reads:

$$
\Psi_{3}=N\left(\begin{array}{c}
c p_{3} / E_{+} \\
c p_{+} / E_{+} \\
1 \\
0
\end{array}\right) \psi^{*} ; \quad \Psi_{4}=N\left(\begin{array}{c}
c p_{-} / E_{+} \\
-c p_{3} / E_{+} \\
0 \\
1
\end{array}\right) \psi^{*}
$$

On the other hand, for a particle with a particular momentum $\boldsymbol{p}$ and energy $E$, on temporally quantized form of Dirac equation given in equation 22 with negative sign in terms of spinors:

$$
\left(E-\beta m c^{2}\right) u \psi=\alpha^{i} p_{i} c u \psi^{*}
$$


which in terms $\alpha^{i}$ and $\beta$ given in equation 35 read,

$$
\left(\begin{array}{cc}
E-m c^{2}\left(\begin{array}{cc}
1 & 0 \\
0 & 1
\end{array}\right) \psi & -\sigma^{i} p_{i} c \psi^{*} \\
-\sigma^{i} p_{i} c \psi^{*} & E+m c^{2}\left(\begin{array}{ll}
1 & 0 \\
0 & 1
\end{array}\right) \psi
\end{array}\right)\left(\begin{array}{l}
u_{A} \\
u_{B}
\end{array}\right)=0
$$

Using the conventional Feynman-Stueckelberg [22] interpretation, changing $E \rightarrow-E$ and three momentum $\boldsymbol{p} \rightarrow-\boldsymbol{p}_{\text {in }}$ the negative energy solution representing the first row,

$$
\begin{gathered}
\left(\begin{array}{cc}
E+m c^{2}\left(\begin{array}{cc}
1 & 0 \\
0 & 1
\end{array}\right) \psi^{*} & -\sigma^{i} p_{i} c \psi \\
-\sigma^{i} p_{i} c \psi^{*} & E+m c^{2}\left(\begin{array}{ll}
1 & 0 \\
0 & 1
\end{array}\right) \psi
\end{array}\right)\left(\begin{array}{l}
u_{A} \\
u_{B}
\end{array}\right)=0 \\
\left(\begin{array}{cc}
E+m c^{2}\left(\begin{array}{ll}
1 & 0 \\
0 & 1
\end{array}\right) & -\sigma^{i} p_{i} c \\
-\sigma^{i} p_{i} c & E+m c^{2}\left(\begin{array}{ll}
1 & 0 \\
0 & 1
\end{array}\right)
\end{array}\right) \Psi=0
\end{gathered}
$$

where the spinors associated with the time-quantized Dirac equation now take the following form:

$$
\Psi=\left(\begin{array}{c}
u_{A} \psi^{*} \\
u_{B} \psi
\end{array}\right)=\left(\begin{array}{c}
v_{A} \\
v_{B}
\end{array}\right)
$$

However as time-quantized equations hold only when integrated over discrete periodic time intervals by making use of the following identities

$$
\int_{-\pi / 2}^{+\pi / 2} e^{-i \theta} d \theta=\int_{-\pi / 2}^{+\pi / 2} e^{i \theta} d \theta, \quad \int_{\pi / 2}^{3 \pi / 2} e^{-i \theta} d \theta=\int_{\pi / 2}^{3 \pi / 2} e^{i \theta} d \theta
$$

can construct a relationship of the form given below which holds only when integrated over angular periods of $\pi$ consisting of $(-\pi / 2 \rightarrow+\pi / 2)$ and $(\pi / 2 \rightarrow 3 \pi / 2)$ : 


$$
\begin{gathered}
\int_{\pi}\left(E+m c^{2}\right) u_{B} \psi d\left(K^{\mu} X_{\mu}\right)=\int_{\pi} \sigma^{i} p_{i} c u_{A} \psi^{*} d\left(K^{\mu} X_{\mu}\right) \\
v_{B}=\frac{c}{\left(E+m c^{2}\right)}\left(\begin{array}{cc}
p_{3} & p_{-} \\
p_{+} & -p_{3}
\end{array}\right) v_{A}
\end{gathered}
$$

Thus for a given $\boldsymbol{p}$, energy $E= \pm \sqrt{p^{2} c^{2}+m^{2} c^{4}}$ and $\Psi^{\dagger} \Psi=2 E$, there are four independent solutions of equation 42 . Therefore the wave functions in normalized forms are,

$$
\Psi_{1}=N\left(\begin{array}{c}
1 \\
0 \\
\left(c p_{3} / E_{+}\right) \\
\left(c p_{+} / E_{+}\right)
\end{array}\right) ; \quad \Psi_{2}=N\left(\begin{array}{c}
0 \\
1 \\
\left(c p_{-} / E_{+}\right) \\
\left(-c p_{3} / E_{+}\right)
\end{array}\right) ; \quad \Psi_{3}=N\left(\begin{array}{c}
\left(c p_{3} / E_{+}\right) \\
\left(c p_{+} / E_{+}\right) \\
1 \\
0
\end{array}\right) ; \quad \Psi_{4}=N\left(\begin{array}{c}
\left(c p_{-} / E_{+}\right) \\
\left(-c p_{3} / E_{+}\right) \\
0 \\
1
\end{array}\right)
$$

With a similar treatment to the equation given in 22 with positive sign

$$
\left(E-\beta m c^{2}\right)\left(\begin{array}{c}
u_{A} \\
u_{B}
\end{array}\right) \psi=-\alpha^{i} p_{i} c\left(\begin{array}{c}
u_{A} \\
u_{B}
\end{array}\right) \psi^{*}
$$

and observing the following set of identities:

$$
\int_{0}^{+\pi} e^{-i \theta} d \theta=-\int_{0}^{+\pi} e^{i \theta} d \theta, \quad \int_{\pi}^{2 \pi} e^{-i \theta} d \theta=-\int_{\pi}^{2 \pi} e^{i \theta} d \theta
$$

Four independent solutions of equation 48 for the wave functions in normalized forms are

$$
\Psi_{1}{ }^{\prime}=N\left(\begin{array}{c}
1 \\
0 \\
\left(-c p_{3} / E_{+}\right) \\
\left(-c p_{+} / E_{+}\right)
\end{array}\right) ; \quad \Psi_{2}{ }^{\prime}=N\left(\begin{array}{c}
0 \\
1 \\
\left(-c p_{-} / E_{+}\right) \\
\left(c p_{3} / E_{+}\right)
\end{array}\right) ; \quad \Psi_{3}{ }^{\prime}=N\left(\begin{array}{c}
\left(-c p_{3} / E_{+}\right) \\
\left(-c p_{+} / E_{+}\right) \\
1 \\
0
\end{array}\right) ; \quad \Psi_{4}{ }^{\prime}=N\left(\begin{array}{c}
\left(-c p_{-} / E_{+}\right) \\
\left(p_{3} / E_{+}\right) \\
0 \\
1
\end{array}\right)
$$




\section{CONSERVATION OF PROBABILITY}

The spinor representation of the Dirac equations embodies the electro dynamic and kinematic properties of the electron like the charge and current density and the components of the spin density and these values can be extracted using $4 \times 4$ gamma matrices of Dirac equation given in equation 21

$$
\sum_{\mu=0}^{3} i \hbar \gamma^{\mu} \partial_{\mu} \Psi-m c \Psi=0
$$

The equation of continuity can be written as

$$
\partial_{\mu} J^{\mu}=\partial_{\mu}\left(\Psi, \gamma^{0} \gamma^{\mu} \Psi\right)=0
$$

and the charge density and current densities can be identified as,

$$
J^{0}=(\Psi, \Psi), \quad J^{i}=\left(\Psi, \alpha^{i} \Psi\right)
$$

Using the first time quantize solution $\Psi_{1}$ given in equation 47

$$
J^{0}=\rho c=E_{+}\left(\begin{array}{llll}
1 & 0 & \frac{p_{3} c}{E_{+}} & \frac{\left(p_{1}-i p_{2}\right) c}{E_{+}}
\end{array}\right)\left(\begin{array}{c}
1 \\
0 \\
\frac{p_{3} c}{E_{+}} \\
\frac{\left(p_{1}+i p_{2}\right) c}{E_{+}}
\end{array}\right)=\left(E+m c^{2}\right)\left(1+\frac{p^{2} c^{2}}{\left(E+m c^{2}\right)^{2}}\right)=2 E
$$

Charge density is a positive definite quantity irrespective of the basis. For the Dirac basis, the current density in the $x$-direction from the first time quantized solution obtained in equation 47 ,

$$
J^{1}=E_{+}\left(\begin{array}{lll}
1 & 0 & p_{3} c / E_{+}
\end{array} \quad\left(p_{1}-i p_{2}\right) c / E_{+}\right)\left(\begin{array}{cccc}
0 & 0 & 0 & 1 \\
0 & 0 & 1 & 0 \\
0 & 1 & 0 & 0 \\
1 & 0 & 0 & 0
\end{array}\right)\left(\begin{array}{c}
1 \\
0 \\
p_{3} c / E_{+} \\
\left(p_{1}+i p_{2}\right) c / E_{+}
\end{array}\right)=2 p_{1} c
$$

The current density in the $y$-direction is, 


$$
J^{2}=E_{+}\left(\begin{array}{llll}
1 & 0 & \frac{p_{3} c}{E_{+}} & \frac{\left(p_{1}-i p_{2}\right) c}{E_{+}}
\end{array}\right)\left(\begin{array}{cccc}
0 & 0 & 0 & -i \\
0 & 0 & i & 0 \\
0 & -i & 0 & 0 \\
i & 0 & 0 & 0
\end{array}\right)\left(\begin{array}{c}
1 \\
0 \\
p_{3} c / E_{+} \\
\left(p_{1}+i p_{2}\right) c / E_{+}
\end{array}\right)=2 p_{2} c
$$

The current density in the z-direction is,

$$
J^{3}=E_{+}\left(\begin{array}{llll}
1 & 0 & \frac{p_{3} c}{E_{+}} & \frac{\left(p_{1}-i p_{2}\right) c}{E_{+}}
\end{array}\right)\left(\begin{array}{cccc}
0 & 0 & 1 & 0 \\
0 & 0 & 0 & -1 \\
1 & 0 & 0 & 0 \\
0 & -1 & 0 & 0
\end{array}\right)\left(\begin{array}{c}
1 \\
0 \\
p_{3} c / E_{+} \\
\left(p_{1}+i p_{2}\right) c / E_{+}
\end{array}\right)=2 p_{3} c
$$

From equations 54 to 57 the equation of continuity can be obtained

$$
\partial_{\mu} J^{\mu}=\partial_{0}\left(\Psi_{1}, \Psi_{1}\right)+\partial_{i}\left(\Psi_{1}, \alpha^{i} \Psi_{1}\right)=\partial_{0}(2 E)+\partial_{i}\left(2 p_{i} c\right)=0
$$

where $\Psi_{1}$ is a solution integrated over a discrete angular period $\pi$. Repeating the above procedure for the remaining integrated time-quantized solutions $\Psi_{2}, \Psi_{3}, \Psi_{4}$ and $\Psi_{1}^{\prime}, \Psi_{2}^{\prime}, \Psi_{3}^{\prime}, \Psi_{4}^{\prime}$ obtained in equations 47 and 50 , the validity of the continuity equation can be shown to hold over discrete angular-intervals of $\pi$, or over discrete temporal intervals of $\pi / \omega$. Therefore a time-quantized continuity equation is

$$
\partial_{\mu} J^{\mu}=\partial_{\mu}\left(\Psi, \gamma^{0} \gamma^{\mu} \Psi\right)=\partial_{0}\left(\Psi_{1}, \Psi_{1}\right)+\partial_{i}\left(\Psi_{1}, \alpha^{i} \Psi_{1}\right)=0
$$

\section{TIME QUANTIZED LINE-ELEMENT ASSOCIATED WITH GRAVITY}

The line element associated with Minkowskian or flat space-time or Minkowskian metric is given by

$$
d s^{2}=g_{\mu \nu} d x^{\prime \mu} d x^{\prime v}=g_{00} d x^{\prime 0} d x^{\prime 0}+g_{i i} d x^{\prime i} d x^{\prime i}
$$

where the metric tensor $g_{\mu \nu}$ in west coast notation is a diagonal matrix with diagonal element $(1,-1,-1,-1)$.

$$
c^{2} d \tau^{2}=g_{00} c^{2} d t^{\prime 2}+g_{i i}\left(u^{\prime i}\right)^{2} d t^{\prime 2} \Rightarrow \frac{1}{\gamma^{\prime}\left(t^{\prime}\right)^{2}}=\left(g_{00} c^{2}+g_{i i}\left(\frac{u^{\prime i}\left(t^{\prime}\right)}{c}\right)^{2}\right)
$$

where $\gamma^{\prime}=d t^{\prime} / d \tau, u^{\prime i}=d x^{\prime i} / d t$. Multiplying the above by $(m / \hbar)^{2}$, 


$$
\left(\frac{m c}{\hbar}\right)^{2}=g_{00}\left(\frac{E}{\hbar c}\right)^{2}+g_{i i}\left(k_{i}^{2}\right)
$$

A set of plane waves $\psi$ and $\psi_{c}$ associated with a particle with rest mass $m$ in constant velocity motion is given by $\psi^{\prime}=e^{-i\left(\omega^{\prime} t^{\prime}-\boldsymbol{k}^{\prime} \cdot \boldsymbol{r}^{\prime}\right)}, \psi_{c}{ }^{\prime}=\psi^{\prime{ }^{*}}$. Therefore the above equation can be written in terms of complementary waves as

$$
\left(\frac{m c}{\hbar}\right)^{2}(\psi, \psi)=-g_{00}\left(\psi^{\prime}, \partial_{0}^{2} \psi^{\prime}\right)-g_{i i}\left(\psi_{c}{ }^{\prime}, \partial_{i}^{2} \psi_{c}{ }^{\prime}\right)=\left|g_{i i}\right|\left(\psi_{c}{ }^{\prime}, \partial_{i}^{2} \psi_{c}{ }^{\prime}\right)-g_{00}\left(\psi^{\prime}, \partial_{0}^{2} \psi^{\prime}\right)
$$

where $g_{i i}=-\left|g_{i i}\right|$. When integrated over discrete-time intervals of $\Delta t$ 'satisfies:

$$
\int_{\Delta t^{\prime}} \omega^{\prime}\left(t^{\prime}\right) d t^{\prime}=\int_{\Delta t^{\prime}} \gamma^{\prime}\left(t^{\prime}\right) \frac{m c^{2}}{\hbar} d t^{\prime}=\int_{\Delta t^{\prime}}\left(g_{00} c^{2}-\left|g_{i i}\right|\left(\frac{u^{\prime \prime}\left(t^{\prime}\right)}{c}\right)^{2}\right)^{-1 / 2} \frac{m c^{2}}{\hbar} d t^{\prime}=\pi
$$

and at discrete time instants corresponding to: $\omega^{\prime}\left(t^{\prime}\right) t^{\prime}=n \pi / 2$.

Therefore, for the line-element associated with general-relativistic effects hold within the timediscrete scheme we proposed in this paper, and gives rise to the following second order relativistic wave-equation with complementary waves:

$$
\left(\frac{m c}{\hbar}\right)^{2} \psi=\left|g_{i i}\right| \partial_{i}^{2} \psi_{c}^{\prime}-g_{00} \partial_{0}^{2} \psi^{\prime}
$$

to which applying the form of temporally quantized solution given in equation 34

$$
\begin{aligned}
\frac{1}{\pi} \int_{\pi}\left(\left(i \sqrt{\mid g_{i i}} \gamma^{i \dagger} \partial_{i} \psi_{c}^{\prime}+i \sqrt{g_{00}} \gamma^{0 \dagger} \partial_{0} \psi^{\prime}\right),\left(i \gamma^{i} \sqrt{\left|g_{i i}\right|} \partial_{i} \psi_{c}^{\prime}+i \sqrt{g_{00}} \gamma^{0} \partial_{0} \psi^{\prime}\right)\right) d\left(K^{\mu} X_{\mu}\right) & =\frac{1}{\pi}\left(\frac{m c}{\hbar}\right)^{2} \int_{\pi}(\psi, \psi) d\left(K^{\mu} X_{\mu}\right)
\end{aligned}
$$

a first order time-quantized solution that satisfies the relativistic wave-equation is obtained as

$$
\sqrt{g_{00}} E \gamma^{0} \psi-\sqrt{\left|g_{i i}\right|} \gamma^{i} p_{i} c \psi_{c}=m c^{2} \psi \Rightarrow \sqrt{g_{00}} E \psi-\sqrt{\left|g_{i i}\right|} \alpha^{i} p_{i} c \psi_{c}=\beta m c^{2} \psi
$$

Using the Schwarzschild metric with $g_{00}=\left(1-2 G M / c^{2} r\right),\left|g_{11}\right|=\left(1-2 G M / c^{2} r\right)^{-1}$ with the line-element 


$$
d s^{2}=\left(1-\frac{2 G M}{c^{2} r}\right) c^{2} d t^{2}-\left(1-\frac{2 G M}{c^{2} r}\right)^{-1} d r^{2}
$$

equation 68 reads :

$$
\beta m c^{2} \psi=\left(1-\frac{2 G M}{c^{2} r}\right)^{1 / 2} E \psi^{\prime}-\left(1-\frac{2 G M}{c^{2} r}\right)^{-1 / 2}\left(\alpha^{i} p_{i} c\right) \psi_{c}{ }^{\prime}
$$

This first order solution reduces to the classical Dirac equation, when the general relativistic effects are ignored by setting $g_{00}=\left|g_{i i}\right|=1$ and by removing the time-quantization condition by setting $\psi^{\prime}=\psi_{c}{ }^{\prime}=\psi$.

\section{CONCLUSION}

By introducing an ansatz, that the energy-momentum anti-commutation holds only over discrete time intervals, and not at every instance which gives a type of time quantization, it is shown that although time is treated as a continuous quantity, but the observables like energy, momentum, force, displacement etc., arising from the relativistic energy-momentum relation are conserved over discrete time-intervals. This suggests that, if one were to measure the energy or the momentum, or other observable quantities associated with the four-vector notation, for a duration which is not exactly an integer multiple of the time-quanta or the chronon associated with a given particle state, then there will be an un-avoidable uncertainty associated with the measurement, which fits well with the known energy-time uncertainty relation in quantum mechanics. Additionally, contrary to Lynds' claim that there aren't really any instances but intervals [10], the present work suggests that it is the observables which are time-discrete, and not the time itself. In light of this interpretation, when Zeno's arrow paradox was revisited within the classical limit we observe that one cannot define the conserved observable physical properties attributed to a particle, for a given instance but for a discrete time-interval.

Further, this ansatz leads to the concept that the wave-function of a particle in relativistic motion is associated with a pair of complementary waves; giving an interpretation of the KleinGordon equation in terms of complementary waves. This treatment, when applied to the Dirac equation, whose solutions satisfy the Klein-Gordon equation, gave us a first order solution with complementary waves, associated with Dirac matrices, and showed that the relativistic energymomentum relation holds only over discrete temporal intervals. The solutions associated with the Dirac equation, when the temporal quantization rules are applied, were discussed along with their corresponding classical solutions. The validity of the continuity equation is shown to hold within this 'time-discrete' scheme and the probability densities are shown to be positive definite for every temporal instance. Finally, we extended this scheme to a line-element associated with general relativistic effects and derived a second order relativistically quantized wave-equation that incorporates gravity. As the discreteness in quantum mechanics is combined with the lineelement associated with gravity, perhaps, the ansatz presented in this paper could potentially be further investigated as a possible approach to quantum gravity. 


\section{ACKNOWLEDGEMENT}

The first author is deeply indebted to J.A Gunawardena and Chandre Dharmawardana. Their suggestions and continuous encouragement helped him immensely to investigate and further refine the work presented in this paper.

\section{REFERENCES}

[1] R. Lévi, Journal de Physique et le Radium 8 (4) (1927) 182-198.

[2] H. Snyder, Phys. Rev. 71 (1947) 38-41

[3] C.N. Yang, Phys. Rev. 72 (1947) 874.

[4] H. Margenau, The Nature of Physical Reality. (McGraw-Hill, 1950).

[3] P. Caldirola, Lett. Nuovo Cim. 27 (1980) 225-228.

[4] S. Vaknin, Time Asymmetry Revisited (Thesis (Ph. D.), Pacific Western University (Encino, California,1982).

[5] E.H. Suchard, J. of Modern Phys. 4 (2013) 791-806.

[6] William G. Tifft, Astrophysical Journal 206 (1976) 38-56

[7] H.T Elze, Phys. Lett. A310 (2003) 110.

[8] J.F. Barbero, Guillermo A. Mena Marugan, E.J.S Villasenor. Time uncertainty in quantum gravitational systems. arXiv:grqc/0311073, 2003.

[9] J.J Halliwell, The Interpretation of Quantum Cosmology and the Problem of Time. arXiv:grqc/0208018, 2002.

[10] Hitoshi Kitada, Quantum Mechanical Clock and Classical Relativistic Clock. arXiv:grqc/0102057, 2001.

[11] N.D. George, A. P. Gentle, A. Kheyfets, W.A. Miller, The Issue of Time in Quantum Geometrodynamics arXiv:gr-qc/0302051, 2003.

[12] B. Russell.. Our Knowledge of the External World as a Field for Scientific Method in Philosophy, (Open Court Publishing Co. 1914, Chicago)

[13] P. Lynds. Found. of Phys. Lett. 16 (4) (2003) 343-355.

[14] E. Schrodinger, Annalen der Physik 79 (1926) 361,489.

[15] O. Klein, Z. Phys. 37 (1926) pp. 895-906,

[16] W. Gordon, Z. Phys. 40 (1926-1927) pp. 117-133

[17] D. L. Bulathsinghala, K. A. I. L. Wijewardena Gamalath, ILCPA 9(2) (2013) 103-115.

[18] P. A. M. Dirac, Proc. Roy. Soc. Lond. A 117 (1928) 610-624. 
[19] Aristotle. Physics VI part 9, 239b5-7

[18] T. Aquinas, Commentary on Aristotle's Physics. (Dumb Ox Books, Trans. By R.J. Blackwell, R.J. Spath, W.E. Thirlkel, 1999)

[19] H. Reichenbach. The Philosophy of Space and Time. (Dover Pub.inc. U.S.A, 1958)

[20] L.I. Mandelshtam, I.E. Tamm Jour. phys. (USSR) 9 (1945) pp. 249-254.

[21] P.A.M Dirac, Proc. R. Soc. Lond. A 133, (1931) 60-72.

[22] E.C.G Stueckelberg, Helvetica Physica Acta 14 (1941) pp. 51-80. 